I. Sánchez-Berriel, L.M. Moreno de Antonio

Department of Computer Science and

Systems (Universidad de La Laguna)

E. García Martín, A. Hernández Hernández, B. González González,

A. Cabrera Díaz, J. Alberto

School of Engineering and Technology (ULL)

G. Yanes, R. Betancor-Villalba,

J.J. Hernández

Instituto Canario de Estadística.

\section{Experiences in data visualization of public statistics from the Canary Islands on mobile device}

This poster outlines the experiences in the development of interactive tools for the visualization of public statistics open data. The developed prototypes have been made for web and mobile devices and they allow displaying statistical data collection by the Canary Islands Statistics Institute (ISTAC), both: numerically and graphically. These applications facilitate the analysis of economic, social and environmental variables of the Autonomous Community of the Canary Islands. For this has been used ISTAC BASE, the main system for the storage and spreading of statistics of the Autonomous Community of the Canary Islands. Usually, the statistical analysis is used as a resource for the decision making related to the scope from which the data come from. The purpose of the visualization is to represent them graphically in a clear and precise way, to help in their interpretation, even for an inexperienced public. In all examples, users can select temporal and geographical factors to define their queries. It is important to emphasize that in some cases, aditional sets of indicators have been defined, expanding those from ISTAC, providing users with more information about the reality of society.
All the tools have been designed thinking about its use for the quick and effective consultation of economic, social and environmental data in any location that has internet connection. This characteristic forces us to pay special attention to the usability of the obtained product, so user's tests have been included.

The interest in this subject is due to the limitations imposed by the fact of the reduced dimensions of the screens of the mobile devices. They have allowed design recommendations such as data breakdown, selection of axes, presentation of units, numeric format $o$ and some improvements to facilitate interaction.

Another aspect considered in the design has been the portability to different platforms, reason why all the developments have been realized following strictly the standards of the web. The portability and adaptability of the graphics is therefore a crucial aspect that has been guaranteed with the use of the JavaScript library: D3, based also on HTML, CSS and SVG.

Each of the applications focuses on the following indicators: economic situation, socio-economic indicators at the council level, environmental indicators at the regional level and a personal calculator of the CPI. In the latter case, an API has been developed that allows data to be exposed through a web service for reusing of data by any developer. 


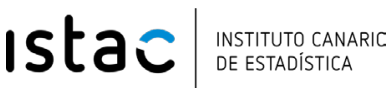

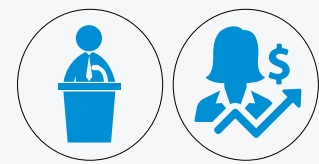

Government and companies

Information is essential in decision making and to measure the efficiency of the taken policies.

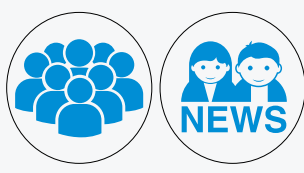

Citizens and media

Each time they demand more data regarding the issues that concern them: economy, environment, population, education, health, etc.

\section{European Statistics Code of Practice}

Principle number 15 refers to the presentation of statistics in a clear and understandable way
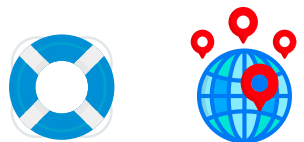

\section{Objectives}

(1) Development of mobile applications to do more easy queries about specific topics

(2) Perform usability tests with users to detect design errors

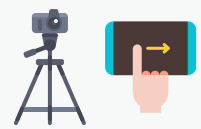

\section{User Experience}

Usability test with users for each application.

Each application up to 7 tasks with 5 users

Operating Systems: Firefox OS, Android User profiles: Statistical experts, computer experts, medium level computer user, low level computer user

\section{Recommendations}

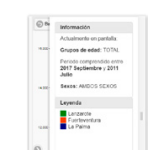

Non-intrusive legends
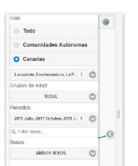

Hierarchical filters

\section{Experiences in data visualization \\ of public statistics from the Canary Islands on mobile device}

\レい $\begin{aligned} & \text { Universidad } \\ & \text { de La Laguna }\end{aligned}$

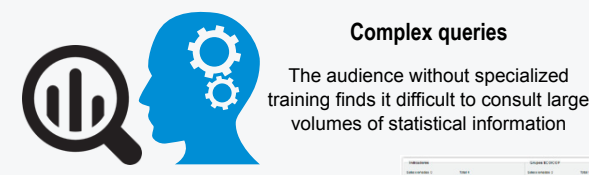

Truthful

Guarantee of data availability

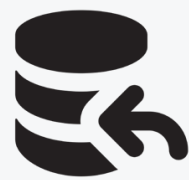

The official statistical agencies are providers of quality socioeconomic data, which offer total reliability

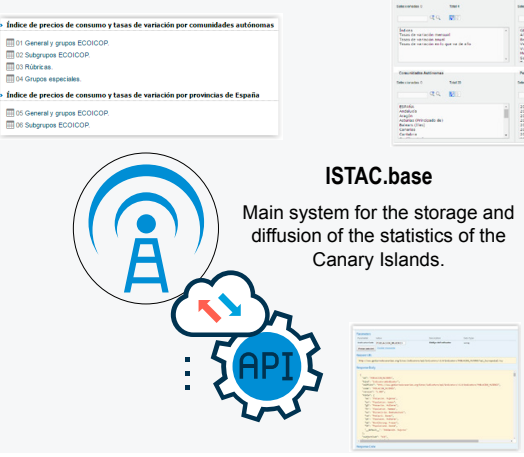

Pulsec app

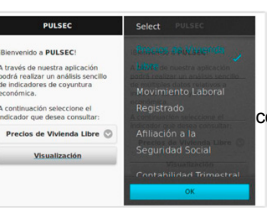

Responsive: Graphics generated using web standards.

Interactive queries: selection lists configured according to the indicato variables

Updated data

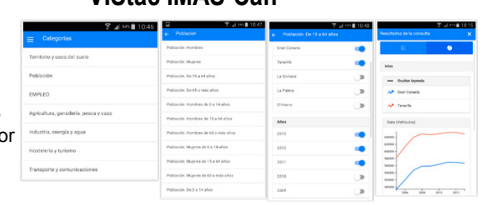

Vistac Situation
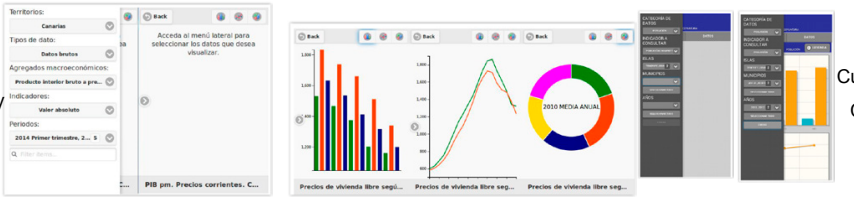

Indicator AP Custom indicators Cross-platform development

\section{Design problems}

The main problems are due to the reduced dimensions of the screen

Tendency to use the mobile in horizontal position

Scarce information in the drawing area

Units are sometimes confusing.

In large series, overflowing of bar plots occurs on the mobile screen.

The user expects information to be placed on the points on the graph

The initial filters generate confusion because a large number of options to be selected

In the screens used for the filters it is expected to find a way to jump to the top of the list
HTML
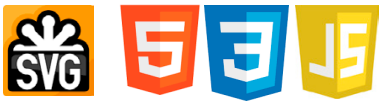

Web standards

\section{Bibliografía}

Castillo, P. N. (2014). Mastering D3. js. Packt Publishing Ltd. Orrù, C., Cuccu, R., Rivolta, G., Sacramento, P., \& Balhar, J. (2017, June).

Multi temporal data visualization in EO mobile apps. In Analysis International Workshop on the (pp. 1-4). IEEE.

UN. Economic Commission for Europe (2009). Cómo hacer (1) MDM__art__Spanish.pd MDM Part2 Spanish pd of Multitemporal Remote Sensing Images (MultiTemp), 2017 9t comprensibles los datos. Parte 1 y Parte 2. Recuperado de: 